\title{
Experimental Investigation of Damage Process in Layer-to-layer Interlock 3D Woven Composites under Uniaxial Tension
}

\author{
Tianhong $\mathrm{Yu}^{1,}$, Shuguang $\mathrm{Li}^{2, \mathrm{~b}}$ \\ ${ }^{1}$ AECC Commercial Aircraft Engine CO.LTD, 3998 South Lianhua RD, \\ Minhang District, Shanghai, 201108, China \\ ${ }^{2}$ Polymer Composite Research Group, B02 ITRC Building, \\ Department of Mechanical, Materials \& Manufacturing Engineering, \\ University of Nottingham, University Park, Nottingham, NG7 2RD, UK \\ atianhongyu_aecc@163.com, bshuguang.li@nottingham.ac.uk
}

Keywords: 3D woven composites, Damage process, Static tensile loading.

\begin{abstract}
The damage behaviour of a type of layer-to-layer interlock 3D woven composite loaded under quasi-static uniaxial tension is studied in this paper. Based on the experimental result obtained, it can be concluded that the development of inter-yarn cracks between warp yarns and the development of through-the-thickness transverse cracks had caused the nonlinear stress-strain responses for loading cases in warp and weft directions respectively. The experimental result provided here may serve as a good reference, based on which a corresponding material damage model may be devised for this type of composite material.
\end{abstract}

\section{Introduction}

Thanks to their superior through-the-thickness material properties when compared to laminated composites and the ease to mould them into structures of complex shapes, 3D textile composites are gaining increasing applications in the aerospace industry. However, before 3D composites of various textile architectures can be utilised for engineering applications, for the interest of structural integrity and safety, it is crucial to understand the damage processes and failure modes associated with these 3D composites.

In this paper, the damage behaviour of a type of layer-to-layer interlock 3D woven composites loaded under quasi-static uniaxial tension is studied. A series of tests for damage inspection have been devised, where samples subjected to different extent of damage were examined using microscopy.

Since only a limited number of studies on damage in layer-to-layer interlock 3D woven composites can be found in the open literature, the experimental investigation presented here also provides an important insight into the complexity of damage development process in this material.

\section{Material and Test Set-up}

For this study, the layer-to-layer interlock 3D woven composite tested was reinforced by E-glass fibres. It was moulded and cured into panels using vacuum assisted resin transfer moulding (VARTM) process with Gurit Prime ${ }^{\mathrm{TM}}$ 20LV infusion epoxy resin. The cured composite has a weft-to-warp ratio of 0.651 (by weight per $\mathrm{cm}$ ) and a total fibre volume fraction of $50.2 \% \pm 0.8 \%$. A CT scan image showing the internal structure of the cured composite is shown in Fig. 1, with axes $\mathrm{X}$ and $\mathrm{Z}$ pointing in the warp and thickness directions respectively. 


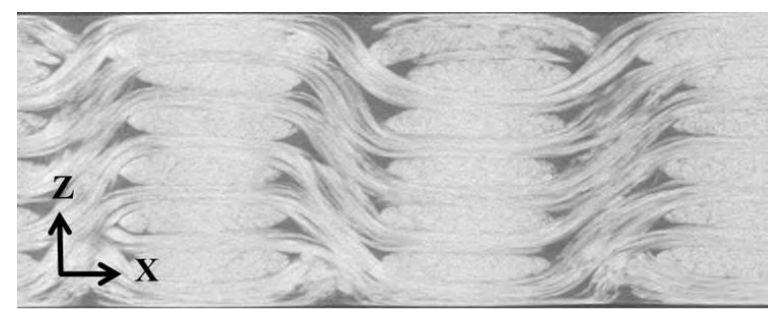

Fig. 1 XZ-plane CT scan image of the cured composite.

Quasi-static uniaxial tensile tests for the warp and weft directions were conducted for the 3D woven composite. The tests were conducted according to ASTM D3039 testing standard [1]. All tensile testing specimens were cut from the composite panels made by the VARTM process mentioned earlier. Dimensions of the specimens are summarised in Table 1.

Table 1. Dimensions of the tensile testing specimens*.

\begin{tabular}{ccccccc}
\hline Test direction & \multicolumn{2}{c}{ Overall length $(\mathrm{mm})$} & Width & $(\mathrm{mm})$ & \multicolumn{2}{c}{ Thickness $(\mathrm{mm})$} \\
\hline Warp direction & 250 & $(1.31)$ & 25.5 & $(0.174)$ & 4.11 & $(0.0309)$ \\
Weft direction & 249 & $(1.24)$ & 25.4 & $(0.112)$ & 4.13 & $(0.0402)$ \\
\hline
\end{tabular}

* Values in "( )" are standard deviation values

The tests were conducted using an electromechanical Instron ${ }^{\circledR} 5985$ universal testing machine, with a $250 \mathrm{kN}$ load cell. A constant testing speed of $2 \mathrm{~mm} / \mathrm{min}$ (cross-head displacement rate) was applied. Load and extension data acquisition rate of $1 \mathrm{kHz}$ was set for all test cases.

Contactless video strain gauge system with an Imetrum ${ }^{\mathrm{TM}}$ standard camera and a telocentric lens was used for strain measurement. Black speckle patterns on top of white coat paint were applied to the specimens, which enabled the video gauge camera to capture local displacement on the specimen surfaces.

Real time acoustic emission (AE) data collection was performed during the tensile tests, along with the stress and strain measurements. The AE data collected serves as a record of sound signals emitted due to material cracking events. As mentioned in [2] and [3], such data can be useful in reflecting different stages of damage development in a specimen.

\section{Warp Direction Tensile Test Result}

Table 2. Warp direction tensile test result.

\begin{tabular}{ccccc}
\hline Property & Mean value & Std. dev. & COV \% & Number of tests \\
\hline Young's modulus (GPa)* & 21.8 & 0.762 & 3.5 & \\
Strength (MPa) & 213 & 13.6 & 6.4 & 7 \\
Ultimate strain \% & 2.05 & 0.311 & 15 & \\
Stress at initial nonlinearity (MPa) & 81.3 & 4.15 & 5.1 & \\
\hline
\end{tabular}

* Values were obtained in the strain range of 500-2500 microstrain 


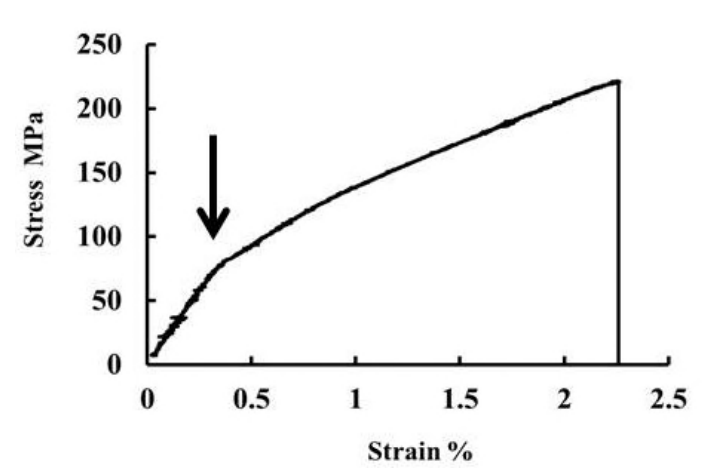

(a) Stress-strain plot

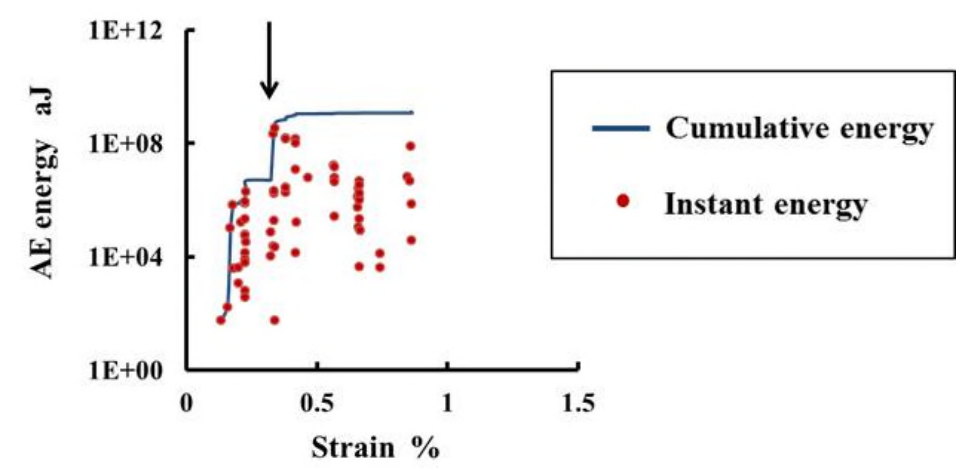

(b) AE data plot

Fig. 2 Typical plots of warp direction tensile test result.

As can be seen from Table 2 and Fig. 2(a), the typical stress-strain behaviour in the warp direction is not linear since there was a sudden degradation of secant modulus (indicated by an arrow in Fig. 2(a)) at around 0.35\% warp direction strain. Viewing Fig. 2(a) in conjunction with Fig. 2(b), one may notice that a step increase in cumulative AE energy also appeared at $0.35 \%$ warp direction strain (indicated by an arrow in Fig. 2(b)), which corresponds to the onset of nonlinearity in the stress-strain curve.

To identify the cause for this nonlinearity of stress-strain response, a number of additional tensile tests were conducted, where a certain degree of damage was introduced into the specimens by loading them in the warp direction until $0.25 \%, 0.4 \%$ and $1 \%$ strains were reached respectively. These specimens were then cut into smaller samples and polished for microscopic examination using optical microscope. By doing so, internal structures of the material before and after reaching the initial point of stress-strain nonlinearity were visually examined, and the damage development in the material was therefore monitored. The microscopic images showing damage in the specimens are presented in Fig. 3.

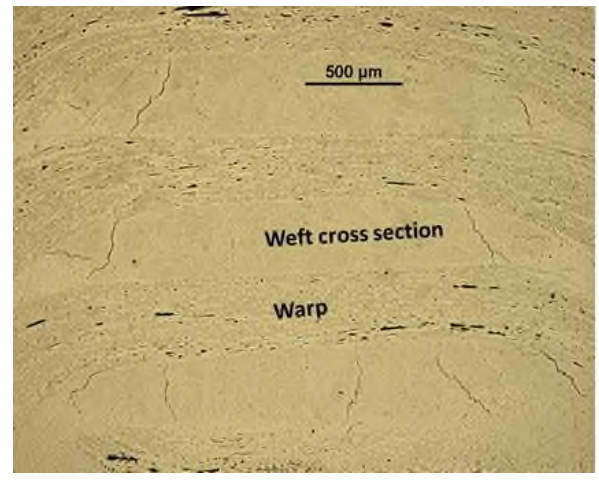

(a) At $0.25 \%$ strain

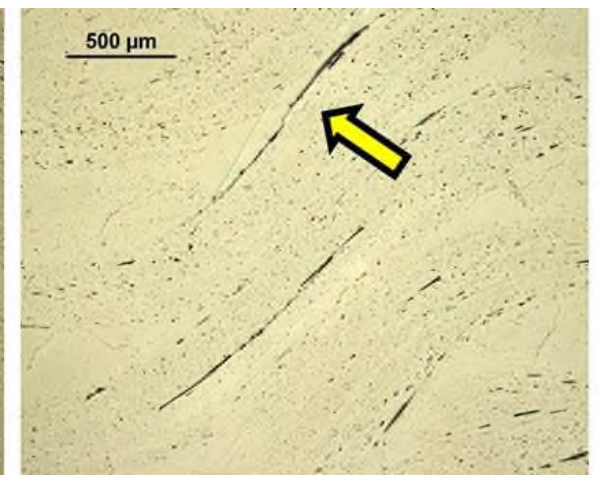

(b) At $0.4 \%$ strain

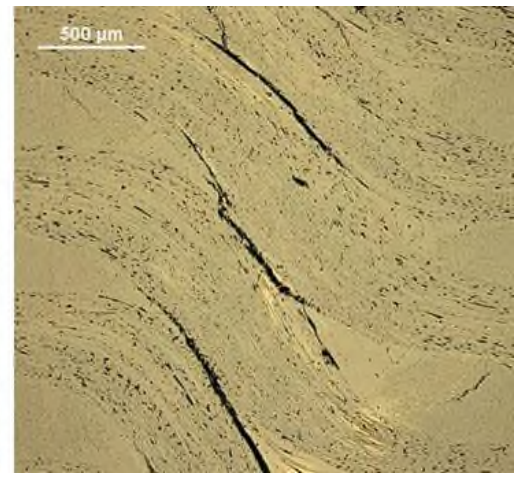

(c) At $1 \%$ strain

Fig. 3 Typical microscopic images of specimens loaded in warp direction tension.

As can be seen in Fig. 3(a), at $0.25 \%$ warp direction strain, before reaching the point of stress-strain nonlinearity $(0.35 \%$ strain $)$, numerous transverse intra-yarn cracks appeared in weft yarns. At this strain level, the stress-strain response was still linear as shown in Fig. 2(a), but substantial amount of AE events were captured as shown in Fig. 2(b). Since no other type of damage was observed at this stage, the development of transverse intra-yarn cracks is considered to be the cause for the AE events recorded before reaching $0.35 \%$ strain.

Then, as can be seen from Fig. 3(b) and Fig. 3(c), when the specimens were further loaded beyond $0.35 \%$ strain, in addition to the intra-yarn cracks, long and increasingly wider inter-yarn cracks appeared between warp yarns at locations where the yarns were curved. Since increase in crack density of intra-yarn cracks was not observed for the specimens loaded beyond $0.35 \%$, it is therefore envisaged that the development of new inter-yarn cracks between warp yarns had caused the onset of stress-strain nonlinearity in Fig. 2(a), as well as the sudden increase of AE energy and 
AE activity in Fig. 2(b). As shown in Fig. 4, the typical final failure of the specimens tested in the warp direction was caused by warp yarn fracture under tension.
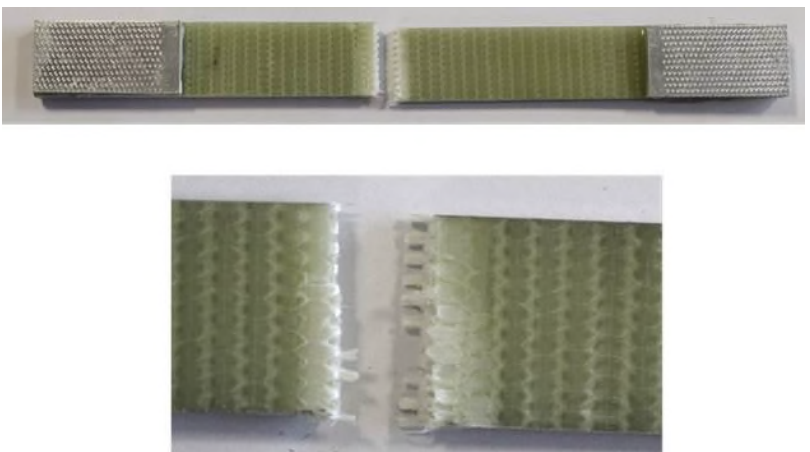

Fig. 4 Typical final failure of specimens tested in warp direction.

\section{Weft Direction Tensile Test Result}

Table 3. Weft direction tensile test result.

\begin{tabular}{ccccc}
\hline Property & Mean value & Std. dev. & COV \% & Number of tests \\
\hline Young's modulus (GPa)* & 25.7 & 1.45 & 5.6 & \\
Strength (MPa) & 337 & 32.6 & 9.7 & 7 \\
Ultimate strain \% & 1.85 & 0.299 & 16 & \\
Stress at initial nonlinearity (MPa) & 94.4 & 8.72 & 9.2 & \\
\hline
\end{tabular}

* Values were obtained in the strain range of 500-2500 microstrain

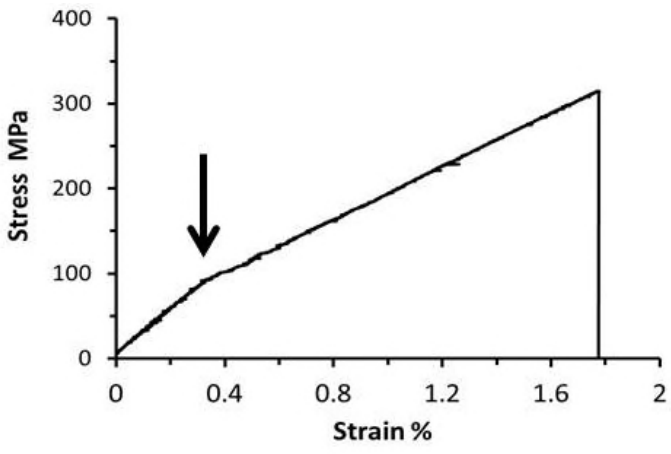

(a) Stress-strain plot

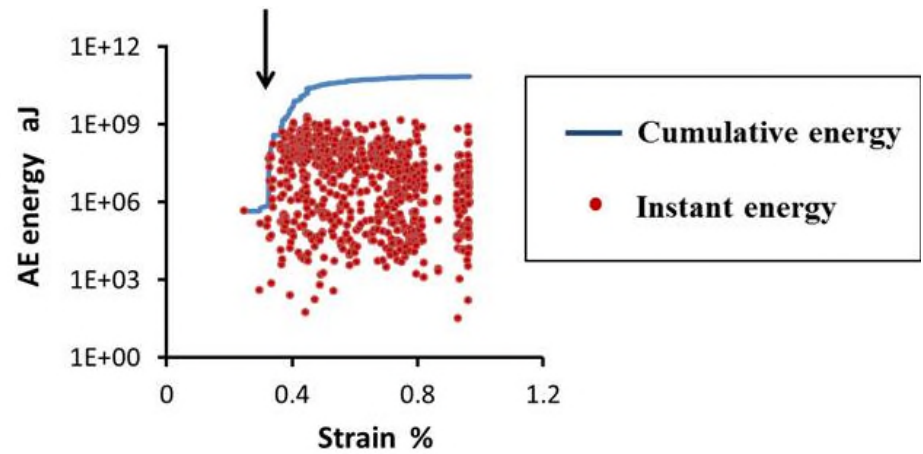

(b) AE data plot

Fig. 5. Typical plots of weft direction tensile test result

As can be seen from Fig. 5(a), similar to the previous case of testing in the warp direction (Fig. 2(a)), for the weft direction, a nonlinear stress-strain curve was once again obtained. Moreover, viewing Fig. 5(a) and Fig. 5(b) in conjunction, it is obvious that a sudden increase of AE energy also correlated well with the onset of stress-strain nonlinearity at around $0.33 \%$ weft direction strain. To investigate the cause for this stress-strain nonlinearity and the sudden increase in AE activity, the same microscopic examination procedure as mentioned earlier was repeated. The microscopic images acquired are illustrated in Fig. 6. 


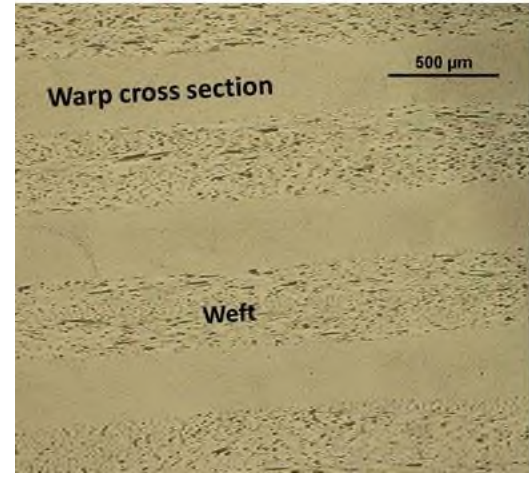

(a) At $0.23 \%$ strain

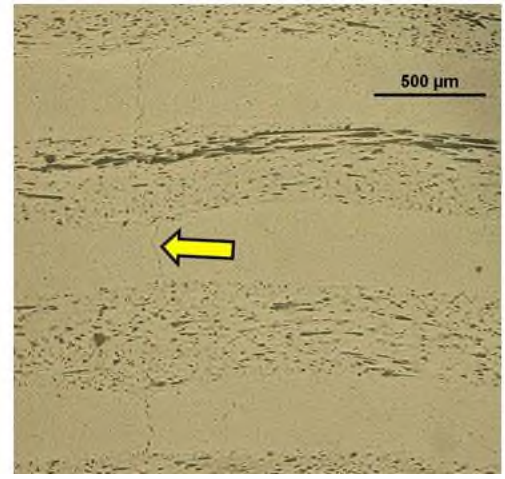

(b) At $0.39 \%$ strain

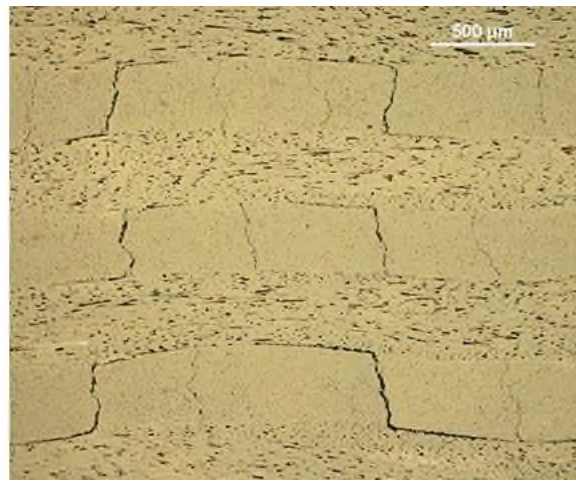

(c) At $0.8 \%$ strain

Fig. 6. Typical microscopic images of specimens loaded in weft direction tension

As shown in Fig. 6(a), before the onset of stress-strain nonlinearity at $0.33 \%$ weft direction strain, there appeared to be no damage in the material. Since there was no crack development at this stage, this explains why nearly no AE event was recorded before $0.33 \%$ weft direction strain (Fig. 5(b)). As the specimens were loaded further, transverse cracks appeared in the warp yarns. As illustrated in Fig. 6(b), at $0.39 \%$ strain, the transverse cracks bypassed weft yarns to form very long cracks extending across the entire thickness of the specimen. By the time $0.8 \%$ strain was reached (Fig. 6(c)), significant increase in crack density was observed for the through-the-thickness transverse cracks, with some of the cracks branched and propagated into boundaries between warp and weft yarns.

With the damage process identified, it is clear that the stress-strain nonlinearity and the sudden increase in AE energy were caused by the development of through-the-thickness transverse cracks. Photographs of the specimens failed under weft direction tension are shown in Fig. 7, where weft yarn fracture and areas of whitening due to extensive matrix cracks can be seen.
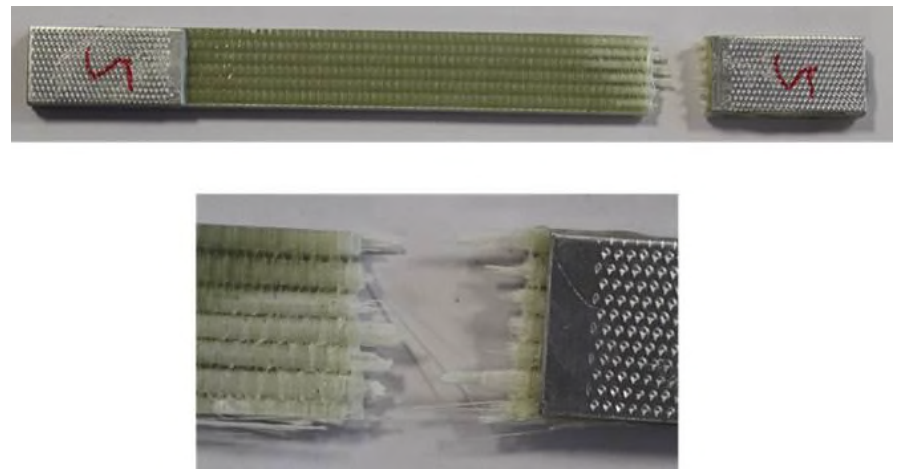

Fig. 7 Typical final failure of specimens tested in weft direction.

\section{Conclusion}

Based on the experimental result presented above, it can be concluded that different damage processes are associated with loadings in warp and weft directions for the layer-to-layer interlock 3D woven composite.

When the 3D woven composite is loaded under tension in the warp direction, the initial damage mode is in the form of intra-yarn transverse cracks in weft yarns, followed by the development of inter-yarn cracks between warp yarns as the material is loaded beyond $0.35 \%$ strain. The resulting nonlinear stress-strain response is in the form of almost bilinear stress-strain curve, which is shown to be caused by the damage effect of inter-yarn cracks.

On the other hand, for the case of tensile loading in the weft direction, through-the-thickness transverse cracks bypassing weft yarns is found to be the main damage mode. This damage is identified to be the cause for the weft direction stress-strain nonlinearity which started beyond $0.33 \%$ strain. 
The experimental result provided here may serve as a good reference detailing the damage behaviour of this novel layer-to-layer interlock 3D woven composite, from which a corresponding material damage model may be built upon and calibrated with.

\section{Acknowledgement}

The financial support provided by AECC Commercial Aircraft Engine CO.LTD, China, through Grant No. RGS106619 to the work presented here is gratefully acknowledged.

\section{References}

[1] ASTM D3039 / D3039M-14, Standard Test Method for Tensile Properties of Polymer Matrix Composite Materials, ASTM International, 2014.

[2] A. E. Bogdanovich, M. Karahan, S. V. Lomov, I. Verpoest, Quasi-static tensile behavior and damage of carbon/epoxy composite reinforced with 3D non-crimp orthogonal woven fabric, Mech. Mater. 62 (2013) 14-31.

[3] S. V. Lomov, D. S. Ivanov, T. C. Truong, I. Verpoest, F. Baudry, K. V. Bosche, H. Xie, Experimental methodology of study of damage initiation and development in textile composites in uniaxial tensile test, Compos. Sci. Technol. 68(12) (2008) 2340-2349. 